\title{
Preserving unitarity in a novel perturbative technique for solving quantum field theory
}

\author{
M. Monoyios \\ Physics Department, Imperial College, London SW7 2BZ, UK
}

Received 10 August 1988; in revised form 30 September 1988

\begin{abstract}
A new perturbative technique for solving a scalar $\varphi^{2 P}$ theory consists of expanding a $\varphi^{2(1+\delta)}$ interaction in powers of $\delta$. The Green functions are computed as a power series in $\delta$ by applying a linear differential operator to the Green functions of a specially constructed intermediate Lagrangian. We confront this linear procedure with the quadratic requirement of perturbative unitarity. We verify up to order $\delta^{3}$ that unitarity is indeed satisfied, by virtue of the precise structure of the intermediate Lagrangian. Unitarity gives constraints on that structure, but does not fix it uniquely.
\end{abstract}

\section{The $\delta$-expansion and unitarity}

A new technique for solving quantum field theory has recently been proposed $[1,2]$ and has been used [3-6] to investigate a variety of problems involving scalar fields. For a $\varphi^{4}$ scalar field theory the method involves writing the interaction as $\varphi^{2(1+\delta)}$ and calculating the Green functions as power series in $\delta$.

To recapitulate the main ideas of $\delta$-perturbation theory (as given in $[1,2]$ ) we consider the following Lagrangian of a scalar field theory in $d$ space-time dimensions.

$$
\mathscr{L}=\frac{1}{2}(\partial \varphi)^{2}-\frac{1}{2} \mu^{2} \varphi^{2}-\lambda M^{2} \varphi^{2}\left(M^{2-d} \varphi^{2}\right)^{\delta}
$$

where $M$ is a mass parameter taking care of the dimensions and $\lambda$ is the dimensionless coupling constant. We expand the interaction in powers of $\delta$, yielding a Lagrangian with logarithmic interaction terms.

$$
\begin{aligned}
\mathscr{L}= & \frac{1}{2}(\partial \varphi)^{2}-\frac{1}{2}\left(\mu^{2}+2 \lambda M^{2}\right) \varphi^{2} \\
& -\lambda M^{2} \varphi^{2} \sum_{k=1}^{\infty} \frac{\delta^{k}}{k !}\left[\ln \left(M^{2-d} \varphi^{2}\right)\right]^{k} .
\end{aligned}
$$

The logarithmic nature of (1.2) appears to rule out the use of a Feynman diagram technique to calculate the Green functions. The problem is overcome by setting up a provisional Lagrangian $\tilde{\mathscr{L}}_{\mathrm{K}}$ given by

$$
\begin{aligned}
\tilde{\mathscr{L}}_{K}= & \frac{1}{2}(\partial \varphi)^{2}-\frac{1}{2}\left(\mu^{2}+2 \lambda M^{2}\right) \varphi^{2} \\
& -\lambda M^{d} \sum_{k=1}^{K}\left(M^{2-d} \varphi^{2}\right)^{\alpha_{k}+1} P_{k} .
\end{aligned}
$$

Here $K$ is an integer specifying the order in powers of $\delta$ to which we wish to calculate the Green functions of the theory in $(1.1,1.2)$, the $\alpha_{k}(k=1,2, \ldots, K)$ are regarded as integers for the present, and the coefficients $P_{k}$ are polynomials in the $\alpha_{k}$ and $\delta$. The form of the $P_{k}$ for general $K$ is not known. The first three sets of polynomials are:

For $K=1$;

$P_{1}=\delta$

For $K=2$;

$P_{1}=\delta+\delta^{2}, \quad P_{2}=-\delta+\delta^{2}$

For $K=3$;

$P_{1}=\delta+\frac{1}{2}\left(1+\alpha_{1}\right) \delta^{2}+\delta^{3}$,

$P_{2}=\omega \delta+\frac{1}{2}\left(\omega^{2}+\alpha_{2}\right) \delta^{2}+\delta^{3}$,

$P_{3}=\omega^{2} \delta+\frac{1}{2}\left(\omega+\alpha_{3}\right) \delta^{2}+\delta^{3}$

where $\omega=\exp (2 \pi i / 3)$.

As the Lagrangian $\tilde{\mathscr{L}}_{K}$ is polynomial in the field $\varphi$ we can develop Feynman rules for computing its Green functions $\widetilde{G}$ to order $\delta^{K}$. We then regard the $\alpha_{k}$ as continuous and act on the functions $\tilde{G}$ with the following differential operator,

$D_{K}=\frac{1}{K} \sum_{j=1}^{K} \sum_{k=1}^{K} \frac{\exp [2 \pi i j(1-k) / K]}{j !}\left(\frac{\partial}{\partial \alpha_{k}}\right)^{j}$

finally setting $\alpha_{1}=\alpha_{2}=\cdots \alpha_{K}=0$. This procedure yields the Green functions $G$ of the theory in (1.1) and (1.2), correct to order $\delta^{K}$.

An interesting question which arises, and which is the subject of this paper, is whether the scattering amplitudes calculated from the Green functions $G$ 
satisfy the unitarity equation to each order in $\delta$. In conventional perturbation theory the scattering amplitudes satisfy, to each order in powers of the coupling constant, an equation reflecting the fact that the $S$-matrix is unitary. For if we write the $S$-matrix as

$\hat{S}=\hat{1}+i \hat{R}$

then the condition that it is unitary yields the following condition on the $R$-matrix elements,

$2 \operatorname{Im} R_{f i}=\sum_{n} R_{f n}^{*} R_{n i}$

where $f, i$ denote the final and initial states respectively, and $n$ labels the possible intermediate states. Equation (1.7) is the unitarity condition which is satisfied by the $R$-matrix elements, or scattering amplitudes, to all orders in weak-coupling perturbation theory. The amplitudes $\widetilde{R}_{f i}$ calculated for the theory in (1.3) are also obtained using conventional Feynman diagram techniques, and consequently they too satisfy (1.7) to each order in $\delta$.

We now note that the left-hand side of $(1.7)$ is linear in the matrix elements of $R$, but that the right-hand side is quadratic. It is this non-linearity which raises the question of whether the scattering amplitudes for the theory in (1.2) satisfy perturbative unitarity, since they are calculated by applying the linear differential operator $D_{K}$ in (1.5) to the $\widetilde{R}_{f i}$. Suppose we calculate the amplitude on the left-hand side of (1.7) as

$R_{f i}^{(K)}=\left.D_{K} \tilde{R}_{f i}^{(K)}\right|_{\alpha_{k}=0}$

where $\widetilde{R}_{f i}^{(K)}$ is calculated from $\widetilde{\mathscr{L}}_{K}$ to order $\delta^{K}$. To obtain an expression of order $\delta^{K}$ on the right-hand side of (1.7) we must compute each of the amplitudes on this side up to order $\delta^{K-1}$ using $\widetilde{\mathscr{L}}_{K-1}$ and $D_{K-1}$. Then the condition that unitarity is satisfied in $\delta$-perturbation theory is that

$$
\begin{aligned}
& 2 \operatorname{Im}\left[\left.D_{K} \tilde{R}_{f i}^{(K)}\right|_{\alpha_{k}=0}\right] \\
& \quad=\sum_{n}\left[\left(\left.D_{k-1} \tilde{R}_{f n}^{(K-1)}\right|_{\alpha_{k}=0}\right)^{* *}\left(\left.D_{K-1} \tilde{R}_{n i}^{(K-1)}\right|_{\alpha_{k}=0}\right)\right]
\end{aligned}
$$

holds to order $\delta^{K}$. This is a non-trivial requirement, since the only constraint on the amplitudes $\widetilde{R}_{f i}$ due to unitarity is that, at order $\delta^{K}$,

$2 \operatorname{Im} \widetilde{R}_{f i}^{(K)}=\sum_{n} \widetilde{R}_{f n}^{(K) *} \widetilde{R}_{n i}^{(K)}$

where all the amplitudes in (1.10) (unlike those in (1.9)) are calculated from $\widetilde{\mathscr{L}}_{K}$, but those on the right-hand side are only given to order $\delta^{K-1}$.

In conventional Feynman graph perturbation theory, (1.7) holds for each diagram which contributes to $R_{f i}$. This leads to a pictorial representation of the unitarity equation, where for each diagram on the left-hand side there exists a so-called cut diagram on the right-hand side, for which slightly different Feynman rules have been invented $[7,8]$, these rules incorporating the summation over intermediate states.

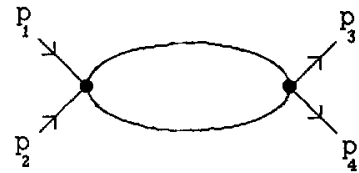

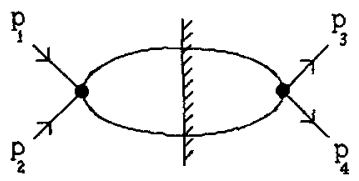

(b)
Fig. 1. a A two-particle scattering process in $\varphi^{4}$ theory. b The cut diagram for the process shown in a

Figure 1a shows a Feynman graph for a scattering process in $\varphi^{4}$ theory, and Fig. $1 \mathrm{~b}$ shows its corresponding cut graph. We shall consider the elastic scattering process in $\delta$-perturbation theory, to show how perturbative unitarity is maintained up to order $\delta^{3}$.

\section{Verification of unitarity up to order $\delta^{3}$}

\subsection{Unitarity to first order in $\delta$}

If we calculate $R$-matrix elements to order $\delta$, the right-hand side of the unitarity equation (1.7) will be of order $\delta^{2}$, and can be neglected in a first approximation. Then unitarity will be satisfied to order $\delta$ provided the imaginary part of $R_{f i}$ is zero to this order.

We first need to consider $\tilde{R}_{f i}$, which is calculated from the intermediate Lagrangian in (1.3), with $K=1$ for a first order computation.

$$
\begin{aligned}
\tilde{\mathscr{L}}_{1}= & \frac{1}{2}(\partial \varphi)^{2}-\frac{1}{2}\left(\mu^{2}+2 \lambda M^{2}\right) \varphi^{2} \\
& -\delta \lambda M^{d}\left(M^{2-d} \varphi^{2}\right)^{\alpha+1} .
\end{aligned}
$$

Here we have relabelled the parameter $\alpha_{1}$ as $\alpha$. The above Lagrangian has one interaction vertex which is proportional to $\delta$, so that the scattering process shown in Fig. 2 will also be of order $\delta$. (See $[2,3]$ for more details of how to evaluate such diagrams.) The contribution to $\tilde{R}_{f i}$ from this diagram is

$$
-\frac{\delta \lambda M^{\alpha(2-\alpha)+2} \Gamma(2 \alpha+3)}{2^{\alpha-1} \Gamma(\alpha)}[\Delta(0)]^{\alpha-1}
$$

where $\Delta(0)$ represents the closed-loop propagator.

The expression in (2.2) is real, which confirms that the theory in (2.1) satisfies perturbative unitarity, and the situation is unchanged when we act on (2.2) with

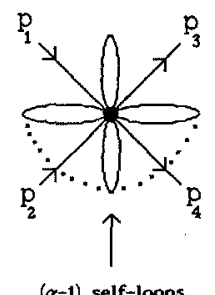

Fig. 2. A two-particle scattering vertex in the theory defined by the Lagrangian in (2.1). The diagram has $(\alpha-1)$ closed loops 
the differential operator in (1.5) with $K=1$, given by

$D_{1}=\frac{\partial}{\partial \alpha}$

and set $\alpha=0$. The quantity obtained is still real, so that unitarity to order $\delta$ is satisfied for the scattering amplitudes of the theory in $(1.1,1.2)$. We note that the diagram in Fig. 2 does not have a corresponding cut graph. This is a reflection of the fact that the imaginary part of the associated scattering amplitude is zero.

\subsection{Unitarity to second order in $\delta$}

To order $\delta^{2}$ the intermediate Lagrangian $\tilde{\mathscr{L}}_{K}$ is given by (1.3) with $K=2$, as

$$
\begin{aligned}
\tilde{L}_{2}= & \frac{1}{2}(\partial \varphi)^{2}-\frac{1}{2}\left(\mu^{2}+2 \lambda M^{2}\right) \varphi^{2} \\
& -\left(\delta+\delta^{2}\right) \lambda M^{d}\left(M^{2-d} \varphi^{2}\right)^{\alpha+1} \\
& -\left(-\delta+\delta^{2}\right) \lambda M^{d}\left(M^{2-d} \varphi^{2}\right)^{\beta+1}
\end{aligned}
$$

where we have put $\alpha_{1}=\alpha$ and $\alpha_{2}=\beta$. We call the two interaction vertices $\alpha$ - and $\beta$-type vertices. The contribution to the left-hand side of the unitarity equation (1.9) comes only from two-vertex diagrams (since the one-vertex diagrams have zero imaginary part, as in the previous section). The diagrams we shall consider are of the type shown in Fig. 3a, with $2 l$ propagators joining the two vertices. (In the final expression for the scattering amplitude we sum all such two-vertex diagrams over the possible values of $l$.) There are four diagrams with $2 l$ lines which we denote by $\widetilde{M}_{\alpha \alpha}, \widetilde{M}_{\beta \beta}, \widetilde{M}_{\alpha \beta}$ and $\widetilde{M}_{\beta \alpha}$, the subscripts labelling the types of vertices in the diagrams. For each of these diagrams there exists a corresponding cut graph of the type shown in Fig. 3b, and unitarity for the theory in (2.4) is satisfied to order $\delta^{2}$ by each diagram, in the conventional way of $(1.10)$.

For instance, the uncut $\alpha \alpha$ graph yields, to order $\delta^{2}$,

$\tilde{M}_{\alpha \alpha}=\delta^{2} \lambda^{2} M^{4} K(l) A\left(p_{1}, p_{2}, p_{3}, p_{4}\right)[F(\alpha, l)]^{2}$

where $A\left(p_{1}, p_{2}, p_{3}, p_{4}\right)$ includes all the factors for the $2 l$ propagators, and where

$$
\begin{aligned}
K(l) & =\frac{1}{\Gamma(2 l+1)}\left[\frac{2}{\Delta(0)}\right]^{2 l} \\
F(\alpha, l) & =\left[\frac{1}{2} M^{2-d} \Delta(0)\right]^{\alpha} \frac{\Gamma(2 \alpha+3)}{\Gamma(\alpha-l+1)} .
\end{aligned}
$$

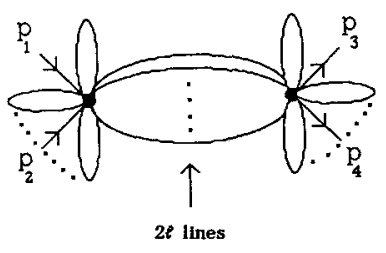

(a) (b)

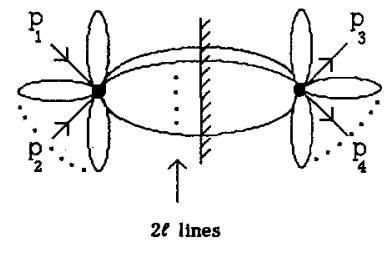

Fig. 3. a A two-particle scattering diagram with $2 l$ propagators. For an $\alpha$-type vertex there are $(\alpha-l)$ closed loops on the vertex. b The cut diagram for the process shown in a
The other uncut graphs of the type in Fig. 3 are given by similar expressions:

$$
\begin{aligned}
\tilde{M}_{\beta \beta} & =\frac{\tilde{M}_{\alpha \alpha}}{[F(\alpha, l)]^{2}}[F(\beta, l)]^{2} \\
\tilde{M}_{\alpha \beta} & =\tilde{M}_{\beta \alpha}=-\frac{\tilde{M}_{\alpha \alpha}}{[F(\alpha, l)]^{2}} F(\alpha, l) F(\beta, l) .
\end{aligned}
$$

The cut graphs yield the same expressions as those in $(2.5,2.7)$ but with $A\left(p_{1}, p_{2}, p_{3}, p_{4}\right)$ replaced by the corresponding expression for the cut propagators, which we call $A\left(p_{1}, p_{2}, p_{3}, p_{4}\right)_{c}$, and unitarity to order $\delta^{2}$ for the theory in (2.4) is satisfied by virtue of the fact that

$2 \operatorname{Im} A\left(p_{1}, p_{2}, p_{3}, p_{4}\right)=A\left(p_{1}, p_{2}, p_{3}, p_{4}\right)_{c}$.

However, if we wish to verify unitarity to order $\delta^{2}$ for the theory in (1.2) we must use the provisional Lagrangian $\widetilde{\mathscr{L}}_{1}$ in $(2.1)$ to calculate each of the amplitudes on the right-hand side of (1.9) to order $\delta$. In other words, there is only one cut graph with $2 l$ propagators, since $\widetilde{\mathscr{L}}_{1}$ has only one type of interaction vertex. In this case, the unitarity equation (1.9) reads

$$
\begin{aligned}
& 2 \operatorname{Im}\left[\left.D_{2}\left(\tilde{M}_{\alpha \alpha}+\tilde{M}_{\beta \beta}+\tilde{M}_{\alpha \beta}+\tilde{M}_{\beta \alpha}\right)\right|_{\alpha=\beta=0}\right] \\
& \quad=\delta^{2} \lambda^{2} M^{4} K(l) A\left(p_{1}, p_{2}, p_{3}, p_{4}\right)_{c}\left(\left.D_{1} F(\alpha, l)\right|_{\alpha=0}\right)^{2}
\end{aligned}
$$

We use (2.5)-(2.8) to reduce the condition (2.9) to

$\left.D_{2}\left((F(\alpha, l)-F(\beta, l))^{2}\right)\right|_{\alpha=\beta=0}=\left(\left.D_{1} F(\alpha, l)\right|_{\alpha=0}\right)^{2}$

and using (1.5) with $K=2$ to give $D_{2}$ we find that (2.10) indeed holds, so that perturbative unitarity is maintained to order $\delta^{2}$.

\subsection{Unitarity to third order in $\delta$}

At order $\delta^{3}$, the Lagrangian $\tilde{\mathscr{L}}_{K}$ in (1.3) with $K=3$ has three types of vertices, which we call $\alpha-\beta$ - and $\gamma$-type vertices. We again consider two-vertex diagrams of the type shown in Fig. 3, but now we must also consider three-vertex diagrams of the type shown in Figs. 4 and 5 , which have $2 l$ propagators joining one pair of vertices, and $2 r$ propagators joining the second pair. (The final expression for the scattering amplitude is obtained by summing over the possible values of $l$ and $r$.) We shall find that the two-vertex and threevertex diagrams satisfy the unitarity relation separately, so that unitarity for the overall scattering amplitude is also maintained.

First we consider the two-vertex diagrams, of which there are nine, distinguished by different types of vertices. We use $\widetilde{\mathscr{L}}_{3}$ to evaluate the nine diagrams to order $\delta^{3}$, which enter the left-hand side of the unitarity equation. We use $\tilde{\mathscr{L}}_{2}$ to evaluate the four cut graphs, which incorporate each amplitude on the right-hand side of the unitarity equation to order $\delta^{2}$. Following the same procedure as in Sect. 2.2 we find that the condition for perturbative unitarity of the theory in 


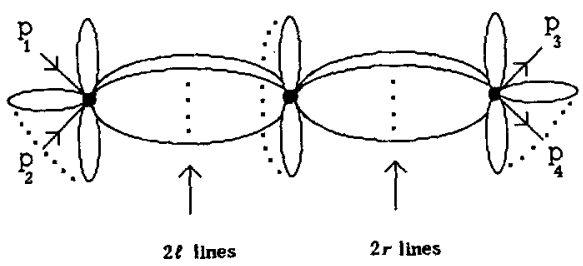

(a)

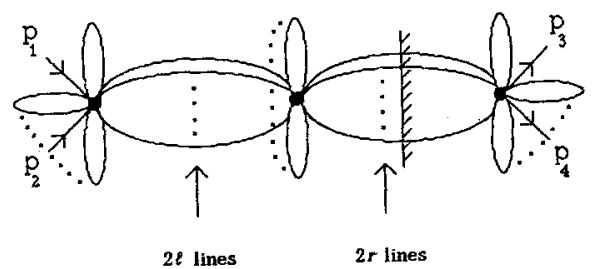

(b)

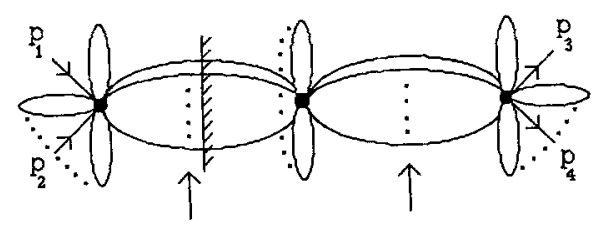

$2 \ell$ lines

$2 x$ lines

(c)

Fig. 4. a A three-vertex scattering diagram in the theory defined by the Lagrangian $\tilde{\mathscr{L}}_{3}$. If the centre vertex is an $\alpha$-type vertex then it has $(\alpha-l-r+1)$ closed loops on it. b,c. The cut graphs for the process shown in $\mathbf{a}$

(1.2) to order $\delta^{3}$ is, for the two-vertex diagrams,

$$
\begin{aligned}
D_{3}[ & \left(a_{11}^{2} \delta^{2}+2 a_{11} a_{12} \delta^{3}\right)[F(\alpha, l)]^{2} \\
& +\left(a_{21}^{2} \delta^{2}+2 a_{21} a_{22} \delta^{3}\right)[F(\beta, l)]^{2} \\
& +\left(a_{31}^{2} \delta^{2}+2 a_{31} a_{32} \delta^{3}\right)[F(\gamma, l)]^{2} \\
& +2\left(a_{11} a_{21} \delta^{2}+\left(a_{11} a_{22}+a_{12} a_{21}\right) \delta^{3}\right) F(\alpha, l) F(\beta, l) \\
& +2\left(a_{11} a_{31} \delta^{2}+\left(a_{11} a_{32}+a_{12} a_{31}\right) \delta^{3}\right) F(\alpha, l) F(\gamma, l) \\
& +2\left(a_{21} a_{31} \delta^{2}+\left(a_{21} a_{32}+a_{22} a_{31}\right) \delta^{3}\right) \\
& +F(\beta, l) F(\gamma, l)]\left.\right|_{\alpha=\beta=\gamma=0} \\
= & \left(\delta^{2}+2 \delta^{3}\right)\left(\left.D_{2} F(\alpha, l)\right|_{\alpha=0}\right)^{2} \\
& +\left(\delta^{2}-2 \delta^{3}\right)\left(\left.D_{2} F(\beta, l)\right|_{\beta=0}\right)^{2} \\
& -\left.\left.2 \delta^{2} D_{2} F(\alpha, l)\right|_{\alpha=0} D_{2} F(\beta, l)\right|_{\beta=0}
\end{aligned}
$$

where $D_{2}, D_{3}$ are given by (1.5) with $\alpha_{1}=\alpha, \alpha_{2}=\beta$, $\alpha_{3}=\gamma$, and the polynomials in (1.4c) for $K=3$ have been written as

$P_{1}=a_{11} \delta+a_{12} \delta^{2}+a_{13} \delta^{3}$,

$P_{2}=a_{21} \delta+a_{22} \delta^{2}+a_{23} \delta^{3}$,

$P_{3}=a_{31} \delta+a_{32} \delta^{2}+a_{33} \delta^{3}$.

We use (1.4) to simplify (2.11) and find that both sides are equal so that the unitarity equation (1.9) is indeed satisfied to order $\delta^{3}$ for the two-vertex graphs.

Now we consider the three-vertex diagrams. There are twenty-seven diagrams of the type shown in Fig. 4a, involving all possible vertex types. We use the Lagrangian $\widetilde{\mathscr{L}}_{3}$ to evaluate these twenty-seven

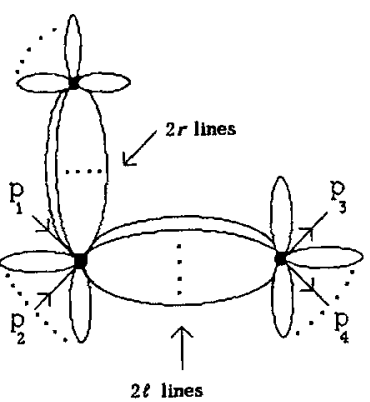

(a)

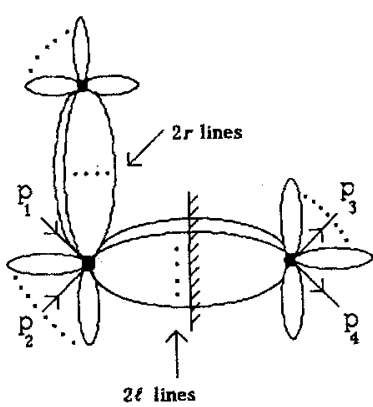

(c)

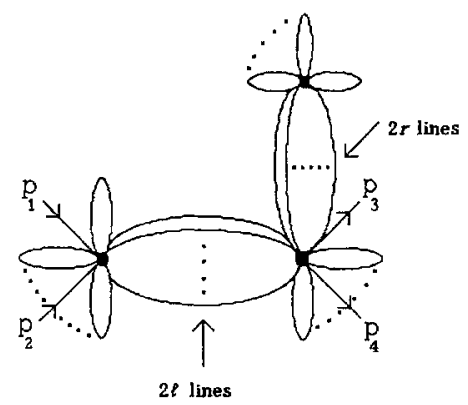

(b)

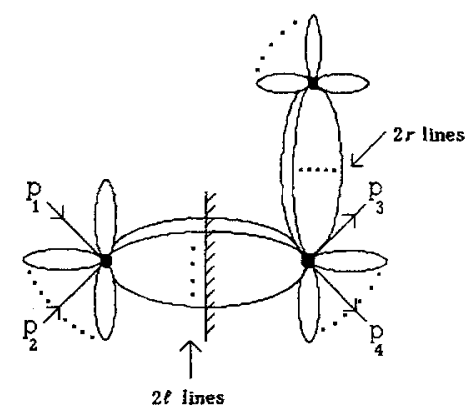

(d)
Fig. 5. a,b Three-vertex scattering diagrams in the theory defined by $\tilde{\mathscr{L}}_{3}$. c The cut diagram for the process shown in a. $\mathbf{d}$ The cut diagram for the process shown in $b$

diagrams to order $\delta^{3}$, and operate on the result with the differential operator $D_{3}$, finally setting $\alpha=\beta=\gamma=0$. This yields

$$
\begin{aligned}
- & \delta^{3} \lambda^{3} M^{6}\left(\frac{1}{2} \Delta(0)\right) K(l) K(r) A\left(p_{1}, p_{2}, p_{3}, p_{4}\right) \\
& -B\left(p_{1}, p_{2}, p_{3}, p_{4}\right) D_{3}\left[a_{11}^{3} V_{\alpha y \alpha}+a_{21}^{3} V_{\beta \beta \beta}+a_{31}^{3} V_{\gamma \gamma \gamma}\right. \\
& +a_{11} a_{21} a_{31}\left(V_{\alpha \beta \gamma}+V_{\alpha \gamma \beta}+V_{\beta \alpha \gamma}+V_{\beta \gamma \alpha}+V_{\gamma \alpha \beta}+V_{\gamma \beta \alpha}\right) \\
& +a_{11}^{2} a_{21}\left(V_{\alpha \alpha \beta}+V_{\alpha \beta \alpha}+V_{\beta \alpha \alpha}\right) \\
& +a_{11}^{2} a_{31}\left(V_{\alpha \alpha \gamma}+V_{\alpha \gamma \alpha}+V_{\gamma \alpha \alpha}\right) \\
& +a_{11} a_{21}^{2}\left(V_{\beta \beta \alpha}+V_{\beta \alpha \beta}+V_{\alpha \beta \beta}\right) \\
& +a_{11} a_{31}^{2}\left(V_{\gamma \gamma \alpha}+V_{\gamma \alpha \gamma}+V_{\alpha \gamma \gamma}\right) \\
& +a_{21}^{2} a_{31}\left(V_{\beta \beta \gamma}+V_{\beta \gamma \beta}+V_{\gamma \beta \beta}\right) \\
& \left.+a_{21} a_{31}^{2}\left(V_{\gamma \gamma \beta}+V_{\gamma \beta \gamma}+V_{\beta \gamma \gamma}\right)\right]\left.\right|_{\alpha=\beta=\gamma=0}
\end{aligned}
$$

where $A\left(p_{1}, p_{2}, p_{3}, p_{4}\right)$ once again includes the factors for the $2 l$ propagators, $B\left(p_{1}, p_{2}, p_{3}, p_{4}\right)$ includes the factors for the $2 r$ propagators, and where we have defined

$$
\begin{aligned}
& V_{x y z}=F(x, l) H(y, l, r) F(z, r) \\
& H(x, l, r)=\left[\frac{1}{2} M^{2-d} \Delta(0)\right]^{x} \frac{\Gamma(2 x+3)}{\Gamma(x-l-r+2)}
\end{aligned}
$$

with $F(x, l)$ defined by (2.6). It is twice the imaginary part of (2.13) which appears on the left-hand side of the unitarity equation.

The cut graphs corresponding to the diagrams represented by Fig. $4 \mathrm{a}$ are of the type shown in Fig. $4 \mathrm{~b}$ 
and $\mathrm{c}$. There are eight diagrams of each type, devised from the possible types of $\alpha$ - and $\beta$-type vertices (recall that we are using $\widetilde{\mathscr{L}}_{2}$ to evaluate the amplitudes on either side of the cut to order $\delta^{2}$, and finding the overall expression for the cut graph to order $\delta^{3}$ ). We operate with $D_{2}$ on the expressions on either side of the cut, and set $\alpha=\beta=0$. When this is done for all sixteen cut graphs we obtain

$$
\begin{aligned}
- & \delta^{3} \lambda^{3} M^{6}\left(\frac{1}{2} \Delta(0)\right) K(l) K(r) \\
& \cdot\left[A ( p _ { 1 } , p _ { 2 } , p _ { 3 } , p _ { 4 } ) B ( p _ { 1 } , p _ { 2 } , p _ { 3 } , p _ { 4 } ) _ { c } D _ { 2 } \left[W_{\alpha \alpha}+W_{\beta \beta}\right.\right. \\
& \left.-W_{\alpha \beta}-W_{\beta \alpha}\right]\left.\left.\right|_{\alpha=\beta=0} D_{2}[F(\alpha, r)-F(\beta, r)]\right|_{\alpha=\beta=0} \\
& +A\left(p_{1}, p_{2}, p_{3}, p_{4}\right)_{c} B^{*}\left(p_{1}, p_{2}, p_{3}, p_{4}\right) \\
& \left.\cdot D_{2}[F(\alpha, l)-F(\beta, l)]\right|_{\alpha=\beta=0} \\
& \left.\left.\cdot D_{2}\left[X_{\alpha \alpha}+X_{\beta \beta}-X_{\alpha \beta}-X_{\beta \alpha}\right]\right|_{\alpha=\beta=0}\right]
\end{aligned}
$$

where

$$
\begin{aligned}
& W_{x y}=F(x, l) H(y, l, r) \\
& X_{x y}=H(x, l, r) F(y, r)
\end{aligned}
$$

and

$$
2 \operatorname{Im} A\left(p_{1}, p_{2}, p_{3}, p_{4}\right)=A\left(p_{1}, p_{2}, p_{3}, p_{4}\right)_{c}
$$$$
2 \operatorname{Im} B\left(p_{1}, p_{2}, p_{3}, p_{4}\right)=B\left(p_{1}, p_{2}, p_{3}, p_{4}\right)_{c} \text {. }
$$

It is the quantity in (2.15) which appears on the right-hand side of the unitarity equation. We simplify (2.13) and (2.15), and using the fact that

$$
\begin{aligned}
2 \operatorname{Im} & A\left(p_{1}, p_{2}, p_{3}, p_{4}\right) B\left(p_{1}, p_{2}, p_{3}, p_{4}\right) \\
= & A\left(p_{1}, p_{2}, p_{3}, p_{4}\right) B\left(p_{1}, p_{2}, p_{3}, p_{4}\right)_{c} \\
& +A\left(p_{1}, p_{2}, p_{3}, p_{4}\right)_{c} B^{*}\left(p_{1}, p_{2}, p_{3}, p_{4}\right)
\end{aligned}
$$

we find that perturbative unitarity for the class of graphs shown in Fig. 4a is indeed satisfied to order $\delta^{3}$.

In the same way we find that unitarity is satisfied for the types of graphs shown in Fig. 5a and b, whose cut diagrams are shown in Fig. 5c and d respectively. Thus perturbative unitarity is satisfied for the complete scattering amplitude in $\delta$-perturbation theory to order $\delta^{3}$, in the manner prescribed by (1.9).

\section{Unitarity as a constraint on the polynomials $\boldsymbol{P}_{k}$}

We have observed that the structure of the differential operator $D_{K}$ and the polynomials $P_{k}$, which is designed to produce the correct $\delta$-expansion for the Green functions of the Lagrangian in (1.2), also preserves the unitarity relation perturbatively. Unlike the differential operator $D_{K}$, the polynomials $P_{k}(k=1,2, \ldots, K)$ are not known for general $K$. We might hope to constrain the form of these polynomials using the requirement of perturbative unitarity.

Consider, for example, the analysis in Sect. 2.3 of unitarity to order $\delta^{3}$ for the two-vertex graphs. Let us suppose that we know the polynomials for $K=2$, but that for $K=3$ our knowledge of them is incomplete, so that they are given by
$P_{1}=\delta+x \delta^{2}+\delta^{3}, \quad P_{2}=\omega \delta+y \delta^{2}+\delta^{3}$,

$P_{3}=\omega^{2} \delta+z \delta^{2}+\delta^{3}$

where $x, y$ and $z$ are not known. Let us further suppose that $x, y$ and $z$ are linear functions of the parameters $\alpha_{1}=\alpha, \alpha_{2}=\beta$ and $\alpha_{3}=\gamma$, that is

$x=x_{0}+x_{1} \alpha+x_{2} \beta+x_{3} \gamma$

$y=y_{0}+y_{1} \alpha+y_{2} \beta+y_{3} \gamma$

$z=z_{0}+z_{1} \alpha+z_{2} \beta+z_{3} \gamma$.

Imposing the unitarity constraint (2.11) yields the following five conditions on the unknown coefficients in (3.2)

$$
\begin{aligned}
& \left(x_{1}+y_{1}+z_{1}\right)+\omega^{2}\left(x_{2}+y_{2}+z_{2}\right)+\omega\left(x_{3}+y_{3}+z_{3}\right)=0 \\
& \left(x_{0}+y_{0}+z_{0}\right)=0 \\
& \left(x_{1}+y_{1}+z_{1}\right)+\omega\left(x_{2}+y_{2}+z_{2}\right)+\omega^{2}\left(x_{3}+y_{3}+z_{3}\right)=0 \\
& \left(x_{0}+\omega^{2} y_{0}+\omega z_{0}\right)+\left(x_{1}+\omega y_{2}+\omega^{2} z_{3}\right)=0 \\
& \left(x_{0}+\omega y_{0}+\omega^{2} z_{0}\right)=\frac{3}{2} .
\end{aligned}
$$

These conditions are not sufficient to fix the polynomials uniquely (and it can be shown that no additional conditions are obtained from considering three-vertex diagrams). The form of the polynomials in (1.4) was originally arrived at using a functional integral representation of the Green functions $G$ of the theory in (1.2), and requiring that $\left.D_{K} \widetilde{G}\right|_{\alpha_{k}=0}=G$, correct to order $\delta^{K}$. If we do this to order $\delta^{3}$, and assume once again that the polynomials are given by $(3.1,3.2)$, we obtain the same conditions as in (3.3), along with the following additional condition.

$\left(x_{1}+y_{2}+z_{3}\right)=\frac{3}{2}$.

Unitarity thus provides powerful constraints, which are indeed satisfied by the polynomials of (1.4). However, additional constraints, and indeed arbitrary choices, are needed in order to arrive at the specific forms used in [1] and [2], and given in (1.4).

Acknowledgements. I would like to thank H.F. Jones for helpful supervision of this work. I acknowledge the receipt of a research studentship from the Science and Engineering Research Council.

\section{References}

1. C.M. Bender et al.: Phys. Rev. Lett. 58 (1987) 2615

2. C.M. Bender et al:: Phys. Rev. D37 (1988) 1472

3. C.M. Bender, H.F. Jones: Imperial College Preprint TP/87-88/28 (1988), to be published in J. Math. Phys.

4. C.M. Bender, H.F. Jones: Imperial College Preprint TP/87-88/11 (1988), to be published in Phys. Rev.

5. H.F. Jones, M. Monoyios: Imperial College Preprint TH/87-88/21 (1988), to be published in Int. J. Mod. Phys.

6. C.M. Bender, K.A. Milton, S.S. Pinsky, and L.M. Simmons, Jr.: Phys. Lett. B205 (1988) 493; C.M. Bender, K.A. Milton: Oklahoma University Preprint 88/0260 (1988)

7. P. Ramond: Field theory: a modern primer. pp. 204-212, Reading, Mass.: Benjamin/Cummings 1981

8. G.'t Hooft, M. Veltman: Diagrammar, in: Particle interactions at very high energies, Part B; D. Speiser et al. (eds.). New York: Plenum Press 1974 